Article

\title{
Investigation of Metal-Organic Framework-5 (MOF-5) as an Antitumor Drug Oridonin Sustained Release Carrier
}

\author{
Gongsen Chen ${ }^{1}{ }^{1}$, Juyuan Luo ${ }^{1}$, Mengru Cai ${ }^{1}$, Liuying Qin ${ }^{1}$, Yibo Wang ${ }^{1}$, Lili Gao ${ }^{1}$, \\ Pingqing Huang ${ }^{1}$, Yingchao Yu ${ }^{1}$, Yangming Ding ${ }^{1}$, Xiaoxv Dong ${ }^{1}$, Xingbin Yin ${ }^{1, *}$ (D) and \\ Jian $\mathrm{Ni}^{1,2, *}$ \\ 1 School of Chinese materia medica, Beijing University of Chinese Medicine, Beijing 102488, China; \\ gongsenchen@163.com (G.C.); luojy0329@163.com (J.L.); cmr199711@163.com (M.C.); \\ qlytcm@163.com (L.Q.); m13164227164@163.com (Y.W.); gll_17@163.com (L.G.); fengxw0802@163.com (P.H.); \\ yyc_zwq@163.com (Y.Y.); dingyangmingbucm@gmail.com (Y.D.); dxiaoxv@163.com (X.D.) \\ 2 Beijing Research Institute of Chinese Medicine, Beijing University of Chinese Medicine, Beijing 100029, China \\ * Correspondence: yxbtcm@163.com (X.Y.); njtcm@263.net (J.N.); Tel.: +86-10-8473-8616 (X.Y.); \\ +86-10-6428-6407 (J.N.)
}

Received: 16 August 2019; Accepted: 13 September 2019; Published: 16 September 2019

\begin{abstract}
Oridonin (ORI) is a natural active ingredient with strong anticancer activity. But its clinical use is restricted due to its poor water solubility, short half-life, and low bioavailability. The aim of this study is to utilize the metal organic framework material MOF-5 to load ORI in order to improve its release characteristics and bioavailability. Herein, MOF-5 was synthesized by the solvothermal method and direct addition method, and characterized by Scanning Electron Microscopy (SEM), X-Ray Diffraction (XRD), Fourier Transform Infrared Spectrometer (FTIR), Thermogravimetric Analysis (TG), Brunauer-Emmett-Teller (BET), and Dynamic Light Scattering (DLS), respectively. MOF-5 prepared by the optimal synthesis method was selected for drug-loading and in vitro release experiments. HepG2 cells were model cells. MTT assay, 4',6-diamidino-2-phenylindole (DAPI) staining and Annexin V/PI assay were used to detect the biological safety of blank carriers and the anticancer activity of drug-loaded materials. The results showed that nano-MOF- 5 prepared by the direct addition method had complete structure, uniform size and good biocompatibility, and was suitable as an ORI carrier. The drug loading of ORI@MOF-5 was 52.86\% $\pm 0.59 \%$. The sustained release effect was reliable, and the cumulative release rate was about $87 \%$ in $60 \mathrm{~h}$. ORI@MOF-5 had significant cytotoxicity (IC50:22.99 $\mu \mathrm{g} / \mathrm{mL}$ ) and apoptosis effect on HepG2 cells. ORI@MOF-5 is hopeful to become a new anticancer sustained release preparation. MOF-5 has significant potential as a drug carrier material.
\end{abstract}

Keywords: MOF-5; Oridonin; Antitumor; HepG2 cells; sustained release

\section{Introduction}

It is expected that cancer will be the main cause of death and the most important obstacle to increasing life expectancy in every country in the world in the twenty-first century [1]. In 2018, there were 18.1 million incident cancer cases and 9.6 million cancer deaths worldwide. Cancer cases increased by $29.5 \%$ between 2006 and 2018. The incidence and mortality rates for lung cancer, breast cancer, prostate cancer, colorectal cancer, stomach cancer, and liver cancer are among the highest [2]. At present, cancer treatment methods are relatively simple and have large side effects. Therefore, it is very necessary to study new anti-tumor active ingredients and drug delivery systems to improve the efficacy and reduce the side effects of cancer treatment. 
Oridonin (ORI) is an active diterpenoid extracted from the Chinese medicinal herb Rabdosia rubescens $[3,4]$ that exhibits potential anticancer, antiinflammation, and antibacterial effects $[5,6]$. Its chemical structure is shown in Figure 1. It has been noted that ORI has antiproliferative and apoptosis-inducing effects on various types of human cancer cells, including lung, liver, colon, pancreatic, and breast cancer $[7,8]$. Studies showed that ORI significantly augmented JQ1-triggered apoptosis in hepatocellular carcinoma (HCC) cells and in HCC cancer stem-like cells. The enhancement of apoptosis by ORI was associated with the mitochondrial apoptosis pathway [9]. However, the great potential for clinical application of ORI is severely limited by its poor aqueous solubility and low bioavailability [10-12]. A variety of strategies such as graphene oxide or nanosuspensions have been attempted to deliver ORI [13-15], but drug loading is generally low, which limits clinical use. And less attention is paid to the effects of release characteristics. Therefore, it is of profound significance to find a suitable drug carrier for ORI to promote its clinical application.

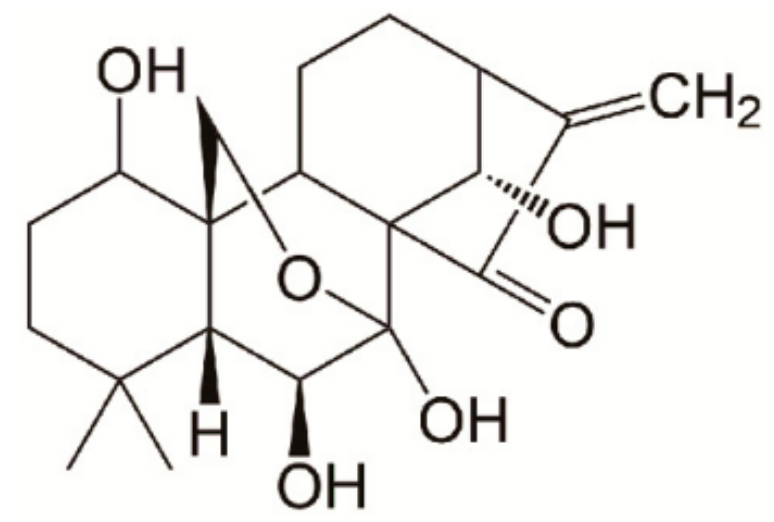

Figure 1. Chemical structure of oridonin.

In recent years, metal organic frameworks (MOFs) have received extensive attention in the application of drug carriers [16,17]. MOFs are highly structured, infinite network structural polymers composed of metal ions and organic ligands [18-20]. As drug carriers, they have the advantages of high pore surface area, adjustable pore diameter, high drug loading, long-term sustained release, good biocompatibility, and complete degradation in vivo [21-24]. Recently, Ke J. and co-workers designed and synthesized a new nitrogen heterocycle organic ligand (2E,2 E)-3,3 -(quinoline-5,8-diyl) diacrylic acid and used it to construct its first Zr-based MOF (termed as ZJU-802). ZJU-802 has been shown to have high anionic drug-loading capacity $(>40 \%)$, low cytotoxicity and good biocompatibility [25].

MOF-5 (also known as IRMOF-1) is one of the most typical representatives of the MOFs family. It is a three-dimensional framework structure composed of terephthalic acid and metal cluster $\mathrm{Zn}_{4} \mathrm{O}$ (Figure 2) [26], firstly synthesized by Yaghi et al. [27]. MOF-5 has open skeleton structure, controlled pore structure and pore surface area and high thermal stability function [28-30], which has been widely studied in gas storage [31] and separation [32], electrochemistry [33], catalysis, and medicine [34]. In particular, the study of MOF- 5 as drug carriers has received much attention in recent years. MOF- 5 was firstly synthesized by solvothermal method, but the current research on MOF- 5 as a drug carrier was mostly synthesized by the direct addition method. The difference between the materials synthesized by the two methods as drug carriers is not yet known. Yang et al. [35] used the direct addition method to prepare MOF-5, which had relatively high drug loading (capsaicin $0.592 \mathrm{~g} / \mathrm{g}$, 5-FU $0.315 \mathrm{~g} / \mathrm{g}$ ), and the release result was good. Liu et al. [36] used the direct synthesis method to prepare MOF-5, and the adsorption rate of curcumin arrived at $94.57 \%$. The hollow structure of MOF-5 was synthesized by Lei $Z$ et al. [37], the drug-loading rate of the modified nanoparticles was $42.43 \%$, which achieved a more ideal sustained release effect. Tetracycline was encapsulated in the Carboxymethylcellulose/MOF-5/GO bio-nanocomposite, which showed effective protection against 
stomach $\mathrm{pH}$ and enhanced the long-time stability of drug dosing [38]. Therefore, as a new drug carrier, MOF-5 has a high drug loading and excellent release properties.

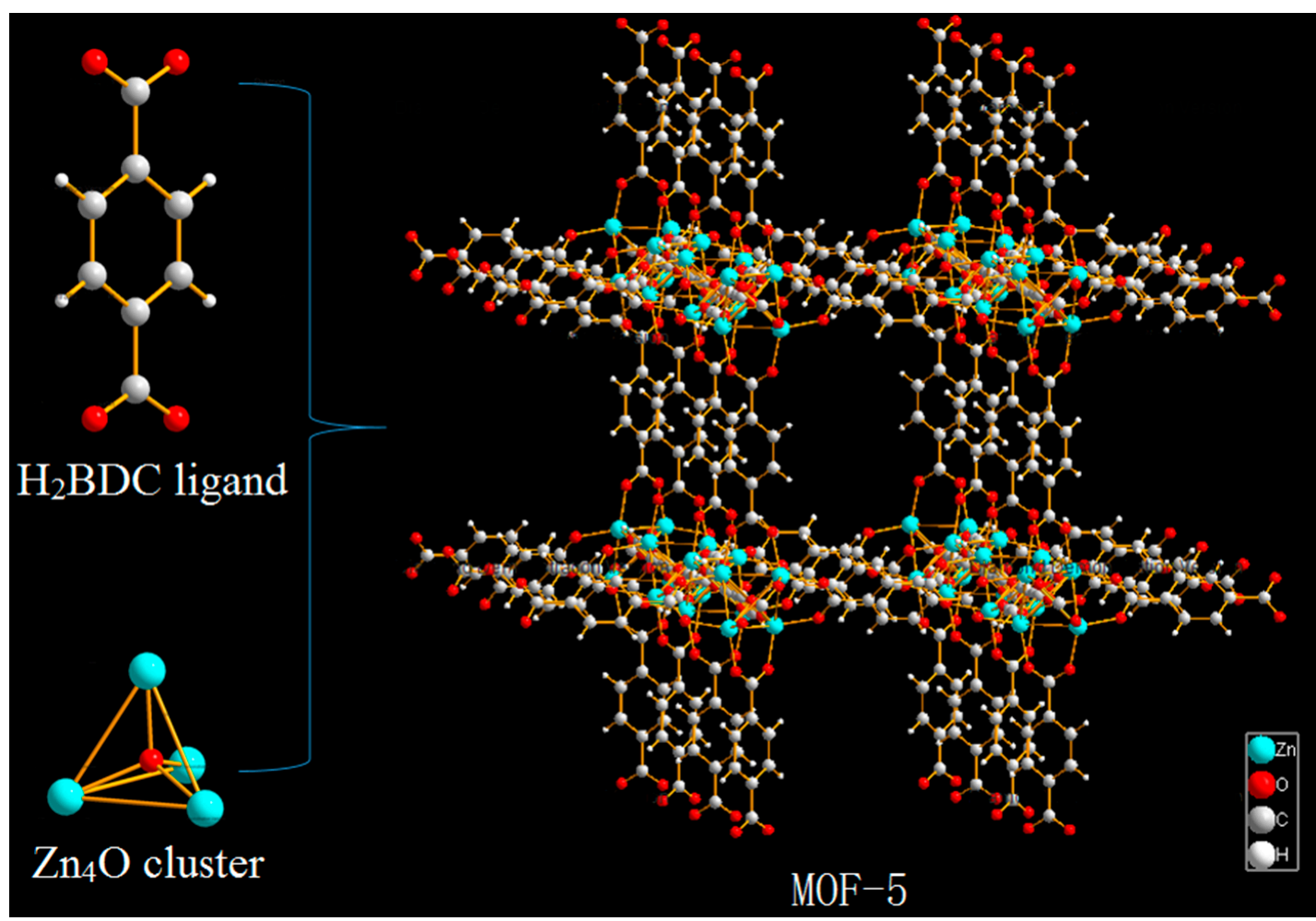

Figure 2. Schematic illustration of the construction of metal organic framework material (MOF-5).

Herein, we synthesized MOF-5 by direct addition approach and solvothermal method, respectively. It was characterized by pharmaceutical methods and carried ORI via solvent adsorption method. The synthesis method which is more suitable for drug carrier was determined by comparison. The in vitro release properties were also examined. The flow chart of synthesis, drug-loading and in vitro release is shown in Figure 3. HepG2 cells were selected as cell models. The biosafety of MOF-5 and the antitumor activity after drug-loading were examined by MTT assay, 4',6-diamidino-2-phenylindole (DAPI) staining and Annexin V/PI assay. Our results indicate that MOF-5, which synthesized by direct addition, has good biocompatibility. It has high drug loading and good sustained release effects as a drug carrier of ORI, and contributes to the anti-tumor effect of ORI. MOF-5 has significant potential as a drug carrier material. 


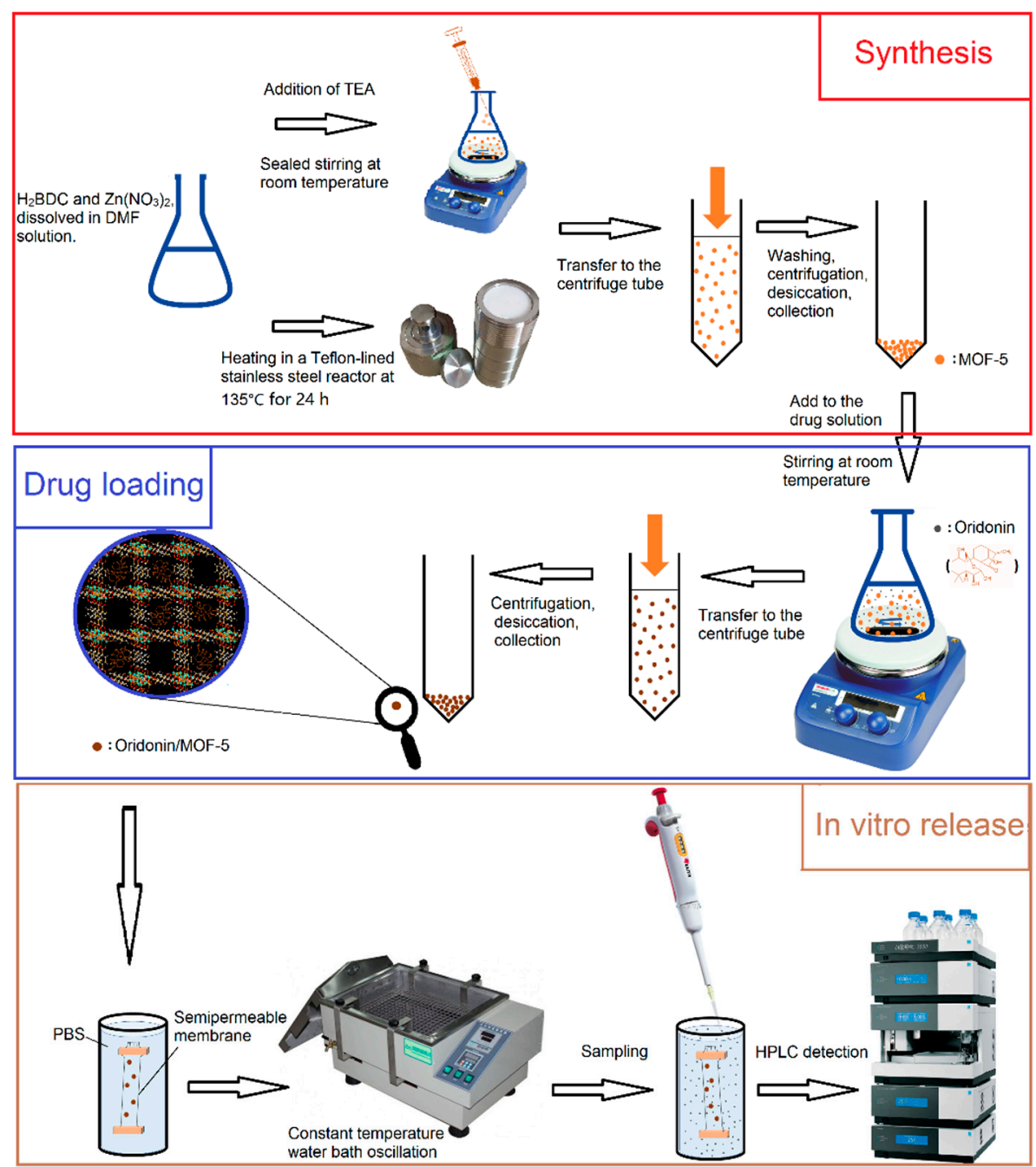

Figure 3. The flow chart for synthesis, drug-loading, and in vitro release.

\section{Materials and Methods}

\subsection{Materials}

Zinc nitrate hexahydrate $\left(\mathrm{Zn}\left(\mathrm{NO}_{3}\right)_{2} \cdot 6 \mathrm{H}_{2} \mathrm{O}\right)$ was purchased from Shanghai Aladdin Biochemical Technology Co., Ltd. (Shanghai, China). Terephthalic acid $\left(\mathrm{H}_{2} \mathrm{BDC}\right)$ and dimethyl sulfoxide (DMSO) were obtained from Tianjin Guangfu Fine Chemical Research Institute (Tianjin, China). Triethylamine (TEA) was acquired from Tianjin Fuchen Chemical Reagent Factory (Tianjin, China). Trichloromethane $\left(\mathrm{CHCl}_{3}\right)$ and $\mathrm{N}, \mathrm{N}$-Dimethylformamide (DMF) were got from Beijing Chemical Factory (Beijing, China). ORI was purchased from Nantong Feiyu Biotechnology Co., Ltd. (Nantong, China). Methanol (Chromatographic reagent grade) was obtained from Thermo Fisher Scientific (Shanghai, China). PBS and penicillin-streptomycin mixture were purchased from Solarbio (Beijing, China). High-glucose Dulbecco's Modified Eagle Medium (DMEM) was obtained from Corning (Manassas, VA, USA). Fetal bovine serum was available from Zhejiang Tianhang Biotechnology Co., Ltd. (Zhejiang, China). 3-(4,5-dimethylthiazol-2-yl)-2,5-dipheny-ltetrazolium bromide (MTT) was obtained from Beijing Biodee Biotechnology Co., Ltd. (Beijing, China). 4',6-diamidino-2-phenylindole (DAPI) and Annexin V-FITC Apoptosis Detection Kit were purchased from Shanghai Beyotime Biotechnology Co., Ltd. (Shanghai, China). All chemicals were of analytical grade or higher. 


\subsection{Characterization}

\subsubsection{Scanning Electron Microscopy (SEM)}

The surface morphology of the synthesized samples was identified using a Hitachi S-4700 cold field emission scanning electron microscope (Tokyo, Japan). High magnification SEM images of MOF-5-S and MOF-5-D can be found in the Supplementary Materials.

\subsubsection{X-Ray Diffraction (XRD)}

X-ray diffraction measurements were investigated through a Rigaku UItima IV X-ray diffractometer (Tokyo, Japan). The measurements were performed using $\mathrm{Cu}-\mathrm{K} \alpha(\lambda=1.541 \mathrm{~nm})$ radiation at $40 \mathrm{kV}$ and $40 \mathrm{~mA}$ in the scan range of $2 \theta$ from 5 to $50^{\circ}$.

\subsubsection{Thermogravimetric Analysis (TG)}

Thermal decomposition was performed using a Mettler Toledo STARe system TGA/DSC3+ thermogravimetric analyzer (Zurich, Switzerland). The samples were heated in alumina pans from $30^{\circ} \mathrm{C}$ to $600{ }^{\circ} \mathrm{C}$ with a heating rate of $10^{\circ} \mathrm{C} / \mathrm{min}$ under $\mathrm{N}_{2}$ flow.

\subsubsection{Fourier Transform Infrared Spectrometer (FTIR)}

The functional group modifications of the particles were detected by a Thermo Fisher Nicolet- 6700 Fourier transform infrared spectrometer (Waltham, MA, USA) at the wavelength range of $400-4000 \mathrm{~cm}^{-1}$.

\subsubsection{Dynamic Light Scattering (DLS)}

The particle size distribution of the samples was measured through dynamic light scattering by a Malvern Nano Zetasizer S90 (Malvern, UK). The particle size distribution was evaluated using pure water as a dispersion medium at room temperature.

\subsubsection{Brunauer-Emmett-Teller (BET)}

The specific surface area and pore size distribution of samples were measured by nitrogen adsorption/desorption at $77 \mathrm{~K}$ using a Belsorp-max BET Sorptometer (Bel Japan Inc., Tokyo, Japan).

\subsection{Preparation of MOF-5}

Direct addition approach: MOF-5 was prepared and activated according to previous reports [39,40]. $\mathrm{Zn}\left(\mathrm{NO}_{3}\right)_{2} \cdot 6 \mathrm{H}_{2} \mathrm{O}(0.62 \mathrm{~g})$ and $\mathrm{H}_{2} \mathrm{BDC}(0.16 \mathrm{~g})$ were fully dissolved in $20 \mathrm{~mL}$ of DMF. And then, $11 \mathrm{~mL}$ of TEA was very slowly injected while stirring. The reaction mixture was sealed and stirred at room temperature for $2 \mathrm{~h}$. After the reaction was done, the solid precipitates were collected by centrifugation, and then washed with DMF and $\mathrm{CHCl}_{3}$ for three times, respectively, and immersed into $\mathrm{CHCl}_{3}$ for 3 days. The solvent was changed once a day. Thereafter, the $\mathrm{CHCl}_{3}$ solvent was decanted and the solids dried at $120^{\circ} \mathrm{C}$ for $12 \mathrm{~h}$ under vacuum. The obtained material was kept in a dryer and named MOF-5-D.

Solvothermal synthesis [41]: The amount of ligand and solvent was unchanged. The reactants were heated in a Shanghai Yushen Teflon-lined stainless-steel reaction vessel (Shanghai, China) at $135^{\circ} \mathrm{C}$ for $24 \mathrm{~h}$, and naturally cooled to room temperature. The post-treatment method was the same as above. The resulting material was named MOF-5-S.

\subsection{Drug-Loading and In Vitro Release Studies}

Solvent adsorption method was used to encapsulate ORI. ORI (90 mg) was dispersed in $6 \mathrm{~mL}$ methanol under ultrasonication. Then, the as-prepared MOF- 5 nanoparticles $(30 \mathrm{mg})$ were immersed in the drug solution and were stirred on a magnetic stirrer with $150 \mathrm{rpm}$ at room temperature for $72 \mathrm{~h}$. The ORI@MOF-5 was centrifuged and washed three times with methanol to get rid of excess ORI. The drug concentration in the supernatant was evaluated by a Thermo Scientific UltiMate 3000 HPLC 
(Waltham, MA, USA). ORI@MOF-5 was dried in the vacuum oven for removing residual methanol. ORI-loading capacity was calculated by using the following equation:

$$
\text { Loading capacity }=\frac{M_{O}-M_{u O}}{M_{O}-M_{u O}+M_{M}} \times 100 \% .
$$

In Equation (1), $\mathrm{M}_{\mathrm{O}}, \mathrm{M}_{\mathrm{uO}}$, and $\mathrm{M}_{\mathrm{M}}$ are the total amount of ORI, the un-loaded amount of ORI in the supernatant, and the amount of MOF-5, respectively.

In vitro release profiles of ORI@MOF- 5 were obtained by the dialysis method. Typically, ORI@MOF-5 was put into dialysis bags and immersed in a certain volume of phosphate buffered saline (PBS) at $\mathrm{pH} 7.4,5.5$ and 2.0. And stirring with $100 \mathrm{rpm}$ at $37^{\circ} \mathrm{C}$ in a SHA-BA Constant temperature bath oscillator (Changzhou, China). Then at certain time intervals, adequate amounts of aqueous solution containing ORI were picked up and replaced with the same amount of fresh buffer. The amount of released drug was measured using HPLC. The corrected concentration and the percent of ORI released were expressed as:

$$
\begin{gathered}
C_{c}=C_{t}+\frac{v}{V} \sum_{0}^{t-1} C_{t}, \\
\text { Drug release }=\frac{M_{R}}{M_{L}} \times 100 \% .
\end{gathered}
$$

In Equation (2), $C_{c}$ stands for the adjusted concentration of ORI at time $t, C_{t}$ is the measured concentration at $t, v$ is the volume of the derived samples, and $V$ is the volume of release solution. $M_{R}$ and $\mathrm{M}_{\mathrm{L}}$ are the amount of released drug and loaded drug (equation 3), respectively.

\subsection{Cell Culture}

HepG2 cells were obtained from Guangzhou Jeniobio Biotechnology (Beijing, China). The cells were cultured in a high-glucose DMEM medium containing with $10 \%$ FBS and antibiotics $(100 \mu \mathrm{g} / \mathrm{mL}$ penicillin and $100 \mu \mathrm{g} / \mathrm{mL}$ streptomycin) for $2-3$ days at $37^{\circ} \mathrm{C}$ under a humid atmosphere with $5 \% \mathrm{CO} 2$. Before passaged, they were harvested by trypsin and resuspended in fresh medium.

\subsection{Cell Viability}

The in vitro cytotoxicity of MOF-5, free ORI and ORI@MOF-5 towards HepG2 cells was assessed by the MTT assay. The 96 -well plates were used to culture HepG2 cells at a density of 5000 cells/well in $100 \mu \mathrm{L}$ overnight. Then, cells were treated with different concentration of MOF-5, free ORI and ORI@MOF- 5 for $24 \mathrm{~h}$. Following, the MTT solutions $(5 \mathrm{mg} / \mathrm{mL}, 20 \mu \mathrm{L})$ were added into each well and further incubated at $37^{\circ} \mathrm{C}$ for $4 \mathrm{~h}$. After that, the medium was discarded and DMSO solvent ( $200 \mu \mathrm{L} /$ well) was added to solubilize the formazan crystals. The absorbance at a wavelength of $490 \mathrm{~nm}$ were measured with a BMG SPECTROstar Nano High-throughput microplate UV spectrophotometer (Ortenberg, Germany) after shaken for $10 \mathrm{~min}$.

\subsection{Nuclear Morphology}

HepG2 cells were cultured at a density of $4 \times 10^{5}$ cells/well in six-well plates and incubated overnight. After treatment with different concentration of MOF-5, free ORI and ORI@MOF-5 for $24 \mathrm{~h}$. The culture supernatant was removed and the cells were fixed with $4 \%$ paraformaldehyde $(500 \mu \mathrm{L})$ for $10 \mathrm{~min}$ at room temperature and then washed twice with PBS. Then cells were incubated with DAPI solution $(10 \mu \mathrm{g} / \mathrm{mL}, 0.8 \mathrm{~mL})$ in the dark for $10 \mathrm{~min}$ and washed twice with PBS again. Morphological changes of nuclei fragmentation and chromatin condensation were photographed using a Nikon Research Inverted Microscope ECLIPSE Ts2R (Tokyo, Japan). 


\subsection{Flow Cytometric for Apoptosis Analysis}

The Annexin V-FITC/PI apoptosis detection kit was used to detect the apoptosis of HepG2 cells by flow cytometry according to the manufacturer's instructions. HepG2 cells were seeded $\left(4 \times 10^{5}\right.$ cells/well $)$ into 6-well plates and incubated overnight. And then incubated with various concentrations of MOF- 5 and ORI@MOF- 5 for 24 h. Following, the cells were harvested and washed with PBS. Then, the cells were suspended in $195 \mu \mathrm{L}$ Annexin V binding buffer, stained with $5 \mu \mathrm{L}$ FITC-labelled Annexin V and $10 \mu \mathrm{L}$ PI, hatching $20 \mathrm{~min}$ in the dark, and then immediately analyzed by a BD FACSCanto II Flow cytometry (Franklin Lakes, NJ, USA).

\subsection{Statistical Analysis}

The statistical analysis was carried out with further statistical methods of single factor analysis of variance, multivariate comparison, and a non-parametric test by using SAS 9.4 software (Beijing, China). Each assay was carried out in triplicate in a parallel manner, and the results were expressed as mean \pm SD. $P$ values $<0.05$ were regarded as significant.

\section{Results}

\subsection{Synthesis and Characterization}

MOF- 5 was synthesized according to the previously described two distinct methods. Before pharmaceutical investigation and biological assays, several techniques were used to characterize the materials, including SEM, XRD, FTIR, TG, and DLS.

The surface morphology of MOF-5-S and MOF-5-D was revealed by SEM and showed in Figure 4A,B. MOF-5-S has a regular hexahedral structure with smooth surface. The particle size distribution is on the order of micrometers, and some of them have angle deficiency. In contrast, MOF-5-D is a nano-sized irregular flaky particle. This may be related to their growth rate. MOF-5-S was slowly synthesized in a high temperature and pressure environment, while MOF-5-D was rapidly grown within a few hours by adding TEA. Therefore, they have obvious differences in appearance and size. SEM images show that prepared MOF-5-D tends to aggregate due to the small size effect caused by water molecules on metal clusters in particles.
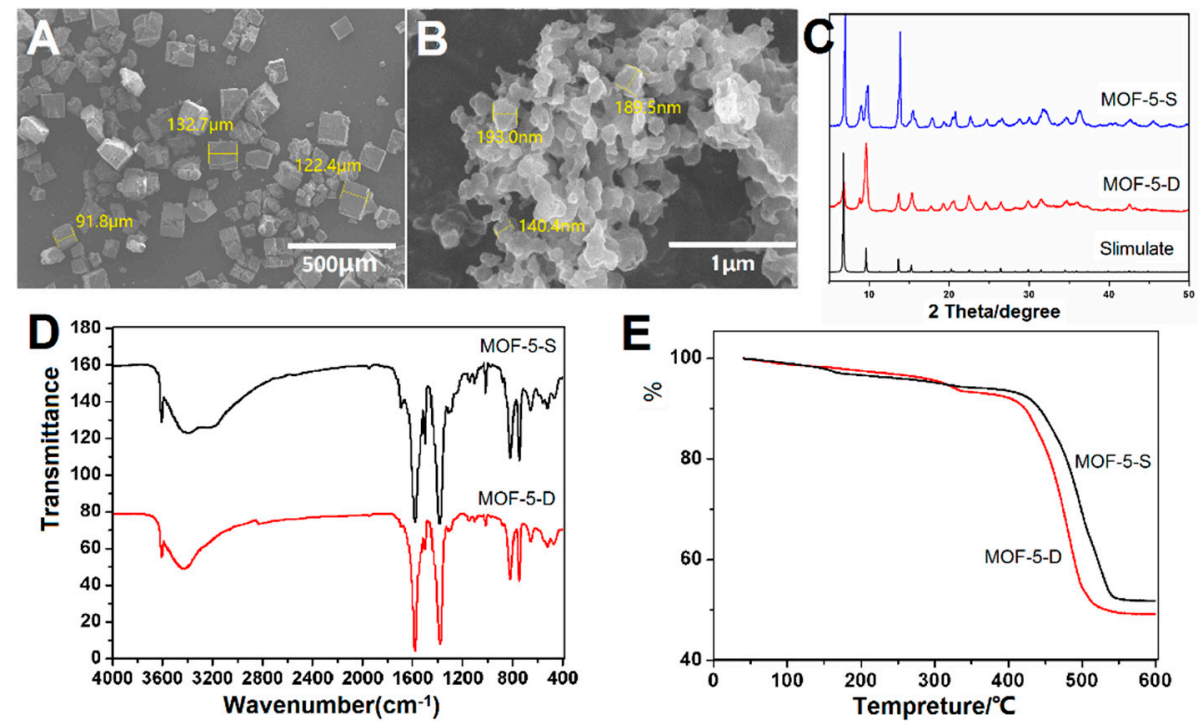

Figure 4. Characterization of MOF-5 synthesized by two methods: (A) Scanning Electron Microscopy (SEM) Image of MOF-5-S; (B) SEM Image of MOF-5-D; (C) X-ray diffraction (XRD) patterns of MOF-5-S and MOF-5-D (D) Fourier Transform Infrared Spectrometer (FTIR) spectra of MOF-5-S and MOF-5-D; (E) Thermogravimetric (TG) analysis of MOF-5-S and MOF-5-D. 
The XRD pattern of the particles has been compared with simulated MOF-5 XRD data as presented in Figure 4C. Acceptable matches are observed for the diffraction peaks of the two sets of samples, which indicates that both methods could synthesize well-structured materials. It has been reported that the intensity of the diffraction peak at $6.8^{\circ}$ is directly proportional to the amount of pore filling material such as solvent [42]. The $6.8^{\circ}$ peak intensity of MOF-5-D is weak in Figure 4C, which showed that most of the solvents in the pore were removed in the post-treatment process. Moreover, diffraction peaks appeared at $8.8^{\circ}$, indicating that the sample may absorb some water, resulting in slight structural changes.

MOF-5-S and MOF-5-D were characterized by FTIR and TG to determine their functional groups, chemical structure and thermal stability. As shown in Figure 4D, the peak shapes and positions in the two spectra are similar, indicating that they have similar functional groups and chemical structures. The two peaks around $800 \mathrm{~cm}^{-1}$ are caused by $\mathrm{C}-\mathrm{H}$ vibration on the benzene ring, which proves that the benzene ring is 1,4-substituted. The strong characteristic peaks at $1590 \mathrm{~cm}^{-1}$ and $1486 \mathrm{~cm}^{-1}$ are the stretching vibration peaks of $-\mathrm{COO}$ bond. The obvious wide absorption peak near $3400 \mathrm{~cm}^{-1}$ corresponds to the absorption peak of water molecules in the sample. The sharp peak at $3610 \mathrm{~cm}^{-1}$ is the combination of metal center with water molecule, which confirms the results of the XRD pattern. The FTIR spectra are consistent with those reported in previous literature [43], indicating that both methods are feasible.

TG analysis (Figure 4E) shows that the thermal stability of the two samples is good and the skeleton is stable before $400{ }^{\circ} \mathrm{C}$. At $400-500{ }^{\circ} \mathrm{C}$, the organic skeleton is decomposed into water and carbon dioxide. A small amount of weight loss before $350{ }^{\circ} \mathrm{C}$ is the detachment of solvent and water molecules inside or outside the pore size of the samples. Finally, the weight is no longer reduced and the residue is $\mathrm{ZnO}$.

The particle size distribution of MOF-5-S and MOF-5-D is shown in Figure 5. As shown in Figure $5 \mathrm{~A}, 87 \%$ of MOF-5-S particles are distributed at $1794 \mathrm{~nm}$, and the remaining small particles may be debris particles. The particle size of MOF-5-D is $260 \mathrm{~nm}$ and it has uniform particle size distribution, as indicated in Figure 5B. $\mathrm{N}_{2}$ adsorption-desorption experiment of MOF-5-S and MOF-5-D was carried out and shown in Figure 5C, and the results are in good agreement with a nano porous materials' structure. The Brunauer-Emmett-Teller (BET) specific surface area of MOF-5-S is $580.35 \mathrm{~m}^{2} / \mathrm{g}$ and the pore volume is $0.2918 \mathrm{cc} / \mathrm{g}$. Those of MOF-5-D are $736.92 \mathrm{~m}^{2} / \mathrm{g}$ and $0.9917 \mathrm{cc} / \mathrm{g}$, respectively. According to BET test results, the specific surface area and pore volume of MOF-5-D are significantly higher than those of MOF-5-S. This may be due to their different synthesis methods. The particle size distribution and BET results showed that MOF-5-D synthesized by direct addition method was more suitable as drug carrier.

Through single factor experiments, ORI was loaded with MOF-5-S and MOF-5-D, respectively, under the same conditions. The loading capacity was compared and the optimum synthesis method was selected. The drug loading of MOF-5-S is $14.23 \% \pm 1.12 \%$, while that of MOF-5-D is as high as $31.36 \% \pm 2.36 \%$. Based on the above experimental results, both methods can successfully synthesize MOF-5. However, the particles synthesized by direct addition method are more suitable as carrier materials for ORI, which will be used for subsequent experiments.

\subsection{Drug Loading}

High drug-loading of drug carriers plays an important role in increasing therapeutic efficiency. Using $\mathrm{L}_{9}\left(3^{4}\right)$ orthogonal experimental design, and three factors affecting drug loading were selected: the ratio of MOF- 5 to ORI, drug-loading time and ORI concentration. The drug loading was $52.58 \% \pm$ $0.59 \%$ (Equivalent to $1.11 \mathrm{~g}(3.04 \mathrm{mmol})$ of ORI per gram of MOF-5) under the optimum conditions: MOF-5: ORI (1:3), 4 days, 15 mg/mL. ORI@MOF-5 was characterized by FTIR and TG, as illustrated in Figure 6. 

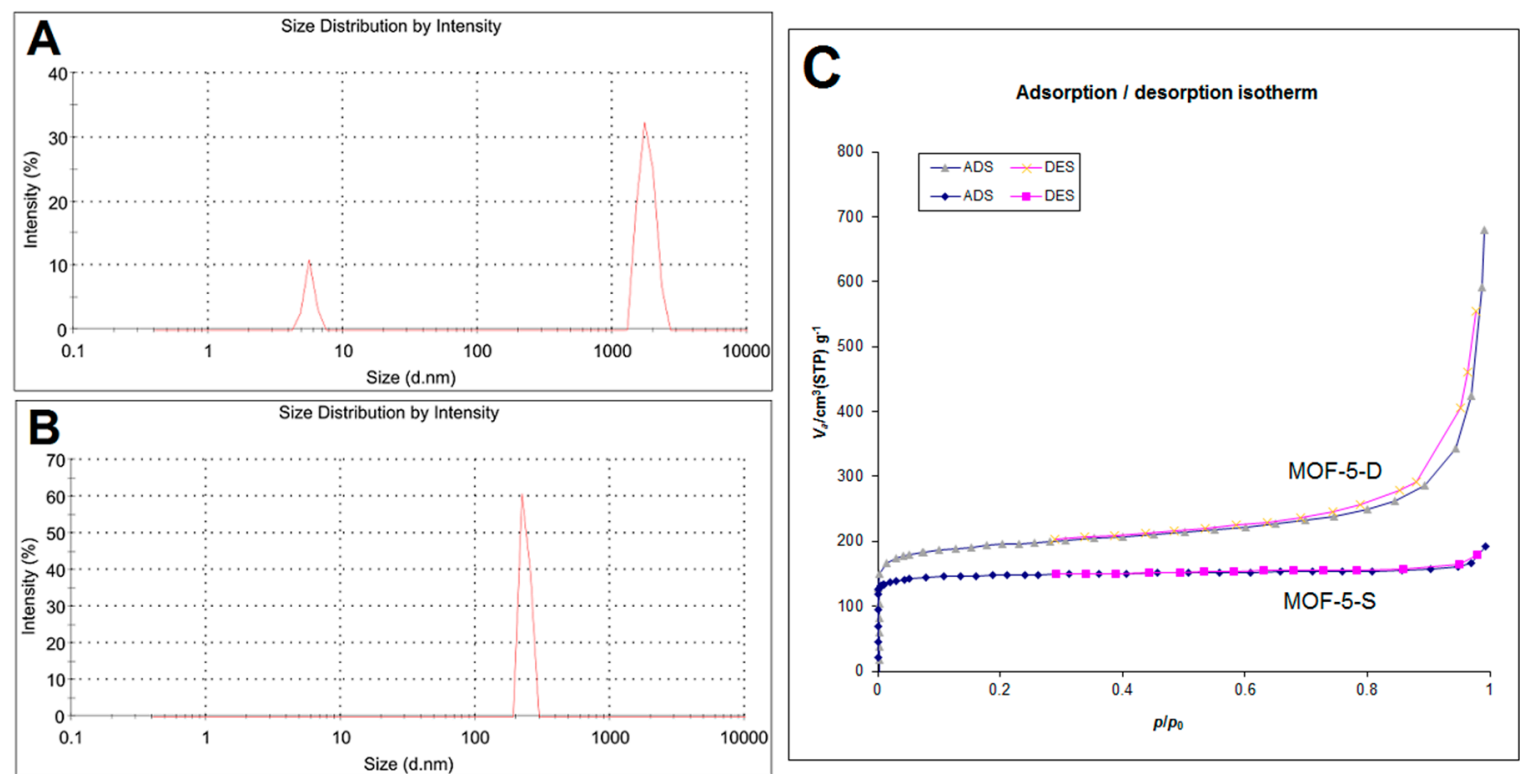

Figure 5. Particle size distribution of MOF-5-S (A) and MOF-5-D (B); (C) Brunauer-Emmett-Teller (BET) isotherm of MOF-5-S and MOF-5-D at $77 \mathrm{~K}$.
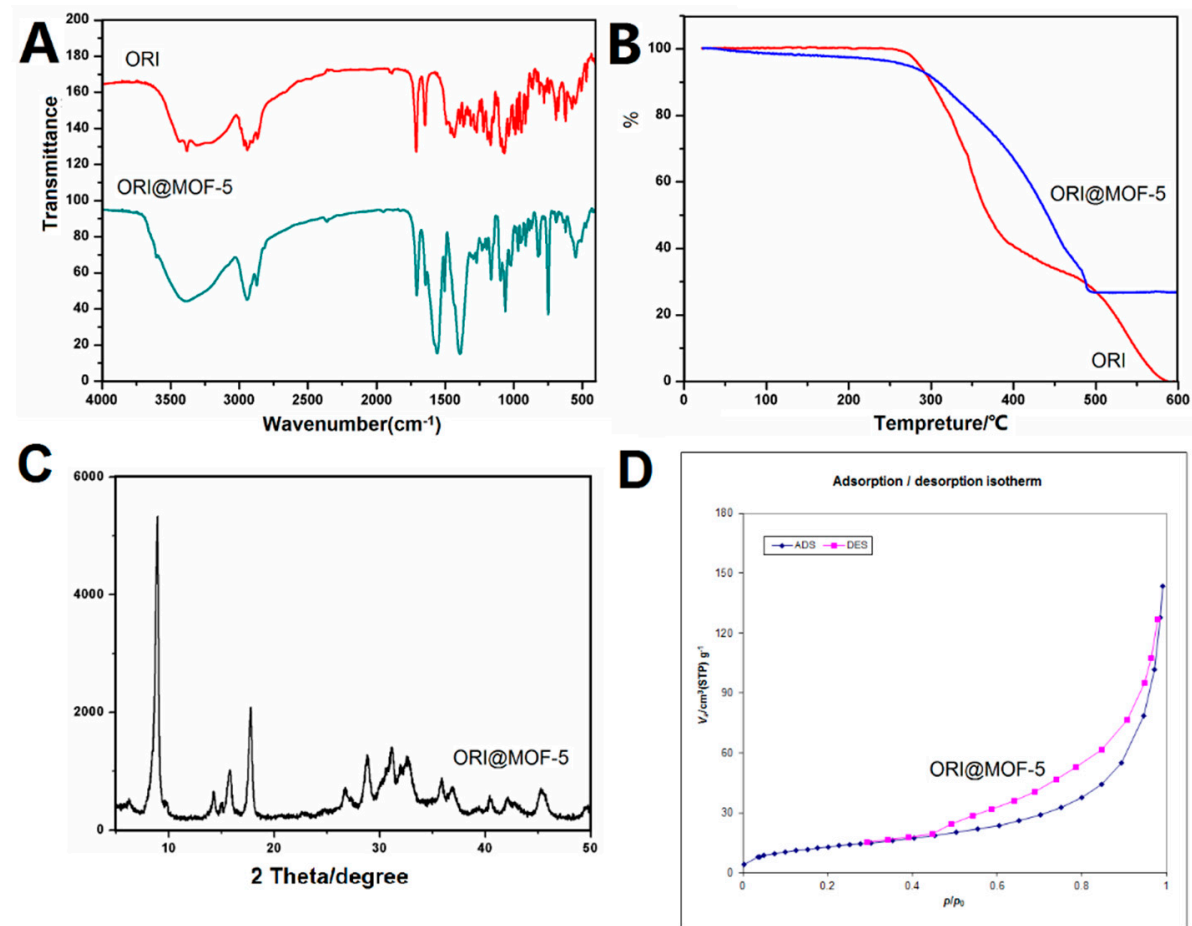

Figure 6. (A) FTIR spectra of ORI and ORI@MOF-5; (B) TG analysis of ORI and ORI@MOF-5; (C) XRD patterns of ORI@MOF-5; (D) BET isotherm of ORI@MOF-5.

It can be obviously seen from the FTIR comparison chart that ORI@MOF- 5 contains not only the characteristic peaks of MOF-5, but also peaks of ORI, such as peaks at $3000-2800 \mathrm{~cm}^{-1}, 1700 \mathrm{~cm}^{-1}$, and $1200-900 \mathrm{~cm}^{-1}$. The result shows that ORI@MOF-5 successfully encapsulates ORI while preserving the structure of MOF-5 itself.

The results of TG analysis show that ORI begins to decompose at $250{ }^{\circ} \mathrm{C}$ and decomposes completely at $600{ }^{\circ} \mathrm{C}$. MOF- 5 mainly loses weight between $400-500{ }^{\circ} \mathrm{C}$. After $500{ }^{\circ} \mathrm{C}$, the weight does not lose any more. ORI@MOF- 5 begins to lose weight at $250{ }^{\circ} \mathrm{C}$ and the weight is no longer reduced at $500{ }^{\circ} \mathrm{C}$, which proves that ORI is successfully loaded. 
The results show that ORI@MOF-5 has good crystallinity. The strong peak at $8.8^{\circ}$ is assumed to be the result of the combination of carbonyl groups and metal centers in ORI. Whether ORI enters the MOF-5 aperture requires BET experiments to reveal.

The BET specific surface area of ORI@MOF-5 is $48.189 \mathrm{~m}^{2} / \mathrm{g}$ and the pore volume is $0.2175 \mathrm{cc} / \mathrm{g}$. They are significantly lower than the blank material, indicating the successful loading of ORI.

\subsection{In Vitro Release}

The in vitro release properties of ORI@MOF- 5 were investigated under different $\mathrm{pH}$ conditions, as shown in Figure 7A. Before 36h, the release rate of ORI@MOF-5 is slightly different under three different $\mathrm{pH}$ conditions, with $\mathrm{pH} 5.5$ being the fastest and $\mathrm{pH} 2.0$ being slower. After reaching 36h, the release rate and total amount of different $\mathrm{pH}$ values tend to be the same. The cumulative release has exceeded $50 \%$ at $12 \mathrm{~h}$. The maximum cumulative release was $87.18 \% \pm 1.26 \%$ at $\mathrm{pH} 7.3,87.02 \%$ $\pm 1.28 \%$ at $\mathrm{pH} 5.5$ and $87.89 \% \pm 0.71 \%$ at $\mathrm{pH} 2.0$. It is indicated that the in vitro release behavior of ORI@MOF-5 is less affected by pH.

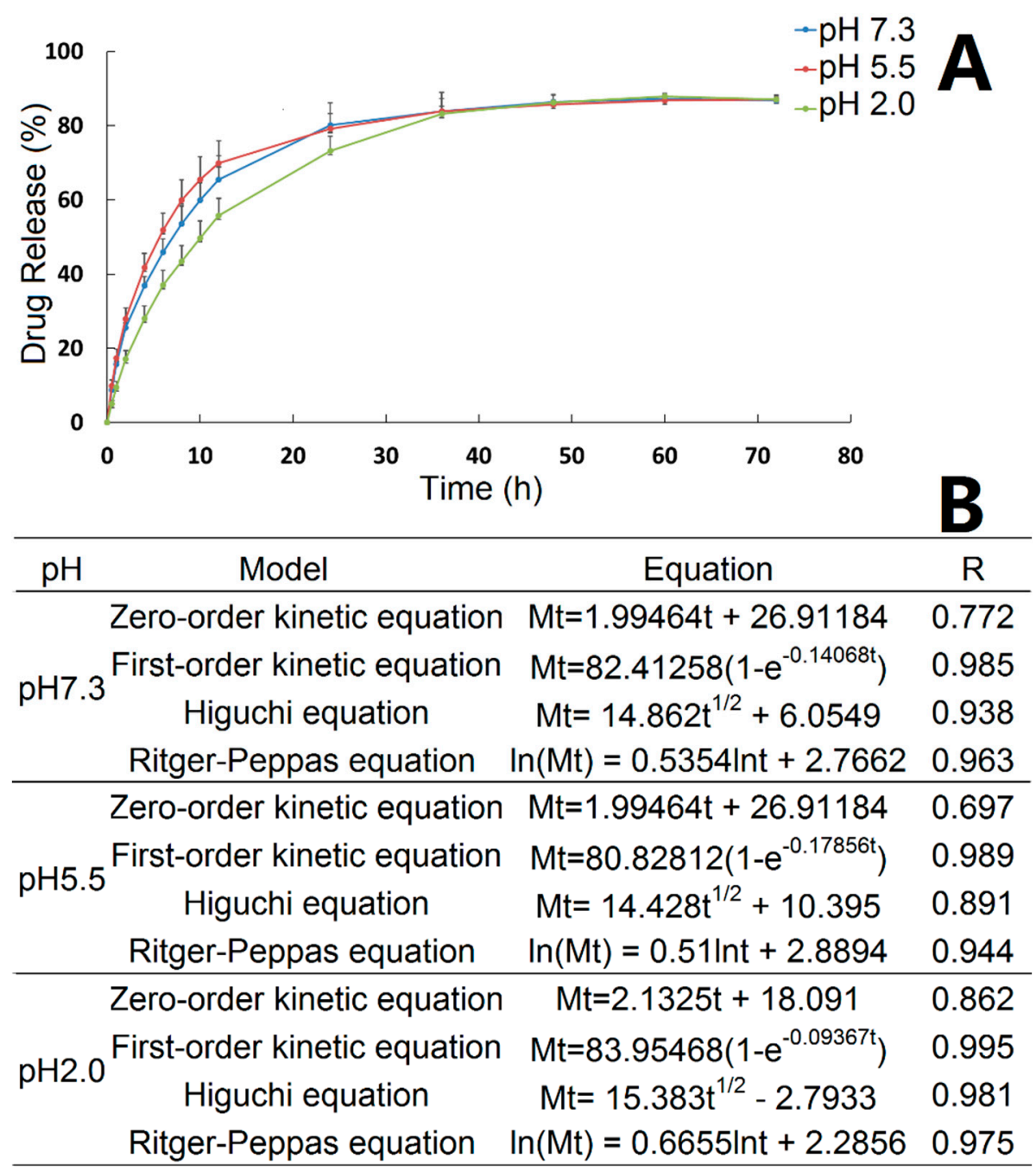

Figure 7. (A) The cumulative release of ORI from ORI@MOF-5 at three $\mathrm{pH}$ values; (B) Fitting results of equation.

Curve fitting was performed on in vitro release results using zero order equation, first order equation, Higuchi equation and Ritger-Peppas equation, as indicated in Figure 7B. In vitro release kinetic study is generally fitted by zero order and first order equations. The release mechanism is usually fitted using the Higuchi equation. However, for the dissolution type skeleton system such as 
MOF material, drugs diffuse from the skeleton material, while the skeleton is also in the dissolution, which accelerates the release of the drug. In this case, there is a substantial deviation in fitting only by Higuchi equation. Peppas et al. proposed an empirical equation that describes the drug release law of the degradable system, named Ritger-Peppas equation. Therefore, we used the zero-order, first-order, Higuchi and Ritger-Peppas equations to fit the release curve for the initial $36 \mathrm{~h}$. Under three $\mathrm{pH}$ conditions, ORI@MOF-5 fits best with the First-order release equation, indicating that the drug-loaded particles have good sustained release effect. For the release mechanism, Ritger-Peppas model fits well. And the diffusion index fitting results of the Ritger-Peppas model, ie, the lnt coefficient, are all between 0.45 and 0.89 , indicating that the release behavior of the particles is consistent with non-Fick's diffusion. ORI@MOF-5 in vitro drug release is the joint result of molecular diffusion and skeleton erosion.

\subsection{Biocompatibility of Nanocarrier}

Before comparing the cytotoxic effects of free ORI and ORI@MOF-5, the biocompatibility of blank nanocarrier should be comprehensively evaluated. Firstly, the classical MTT assay was carried out on the HepG2 cells. According to Figure 8A, the cells reveal high viability after $24 \mathrm{~h}$ incubation of different concentration dosages MOF-5 $(5,10,15,20$, and $25 \mu \mathrm{g} / \mathrm{mL})$, indicating that MOF-5 is non-toxic at the experimental dose.
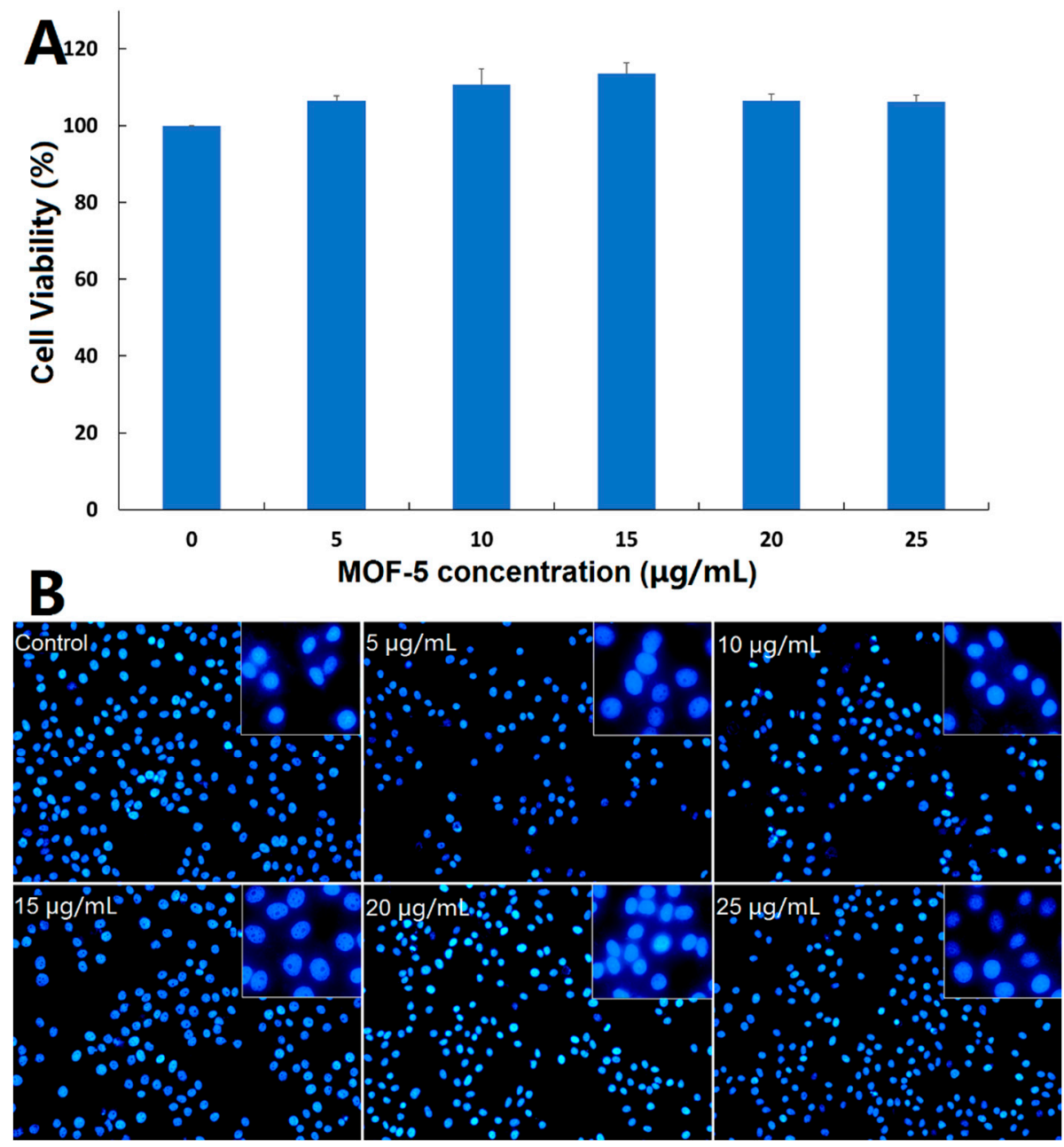

Figure 8. (A) Cytotoxicity study of MOF-5 on HepG2 cells determined by MTT assay; (B) Fluorescent microscopic images of DAPI-stained HepG2 cells following 24-h treatment with different concentrations of MOF-5. 
Then, DAPI staining was used to determine whether MOF-5 had an effect on the nuclear morphology of HepG2 cells. After $24 \mathrm{~h}$ treatments of HepG2 cells with different concentrations of MOF-5, the nucleus morphological of each group are in good shape (Figure 8B), without rupture, deformation, or other phenomena, which confirms the good biocompatibility of MOF-5.

To further verify the cytotoxicity, the Annexin V/PI assay was used to detect the effect of MOF-5 on apoptosis. The results are shown in Figure 9. After being treated with different doses of MOF-5 for $24 \mathrm{~h}$, there is no significant change in the percentage of viable cells, early apoptotic cells, and dead cells. There is no significant induction of apoptosis at the experimental dose, indicating that MOF-5 is biocompatible.
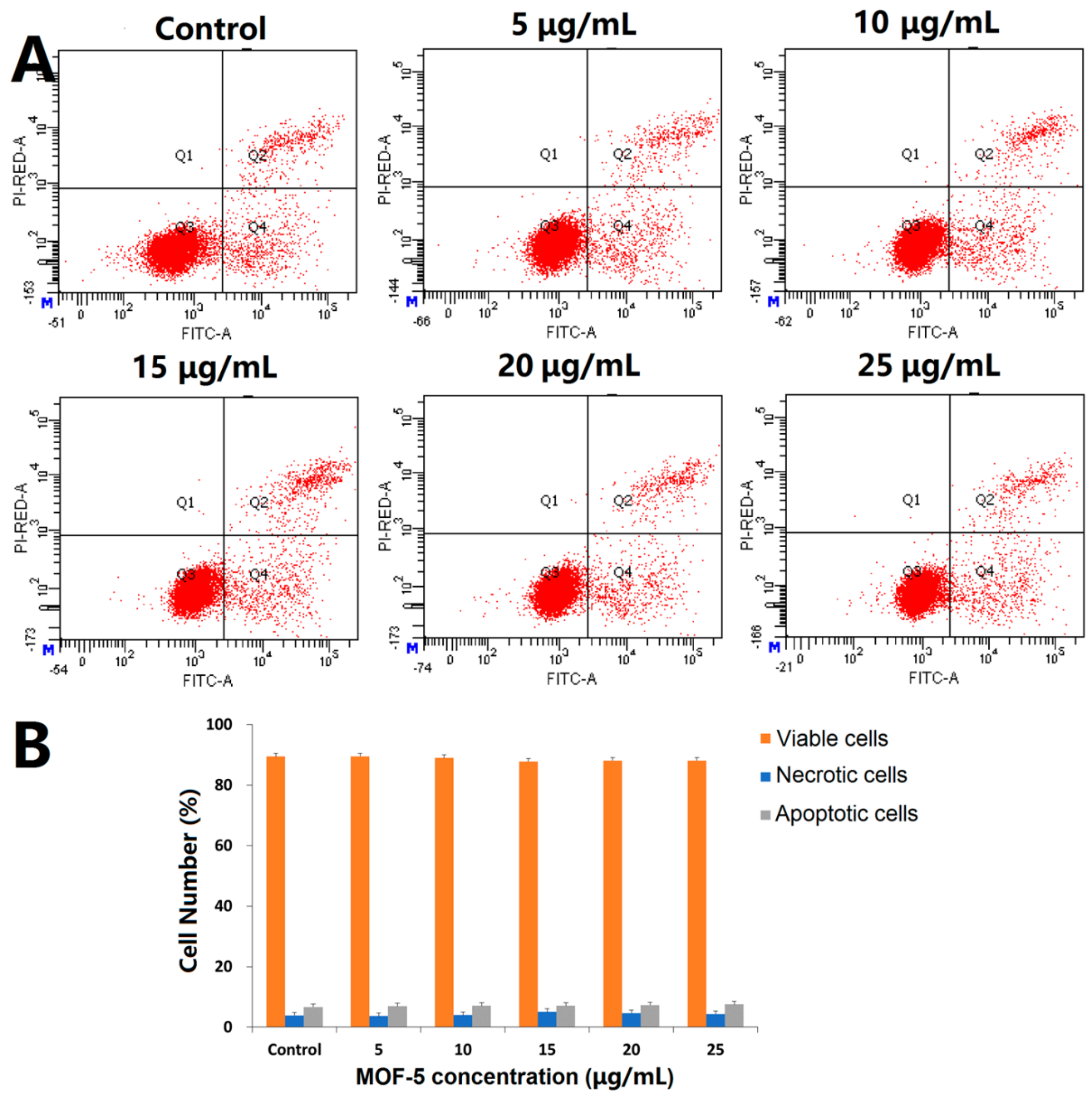

Figure 9. (A) Apoptosis assays for HepG2 cells after treatment with different concentrations of MOF-5 for $24 \mathrm{~h}$; (B) Statistical analysis of cell apoptosis.

\subsection{In-Vitro Toxicity Study of Ori@MOF-5}

HepG2 cells were treated with different doses of free ORI $(5,10,15,20$, and $25 \mu \mathrm{g} / \mathrm{mL})$ and ORI@MOF-5 (Drug loading 52.86\%) containing the same dose of ORI for $24 \mathrm{~h}$, respectively. The results of toxicity comparison by MTT assay are shown in Figure 10A. Cell viability decreases with the increase of drug concentration, indicating that both ORI and ORI@MOF-5 show significant cytotoxicity in HepG2 cells in a dose-dependent manner. At the equivalent ORI concentration, the toxicity of ORI @MOF-5 is slightly lower than that of free ORI. And the IC $_{50}$ of them are ORI: $11.08 \mathrm{ug} / \mathrm{mL}$ and Ori@MOF-5: $22.99 \mathrm{ug} / \mathrm{mL}$, respectively. Analogous results have been reported in previous studies [44,45]. Due to the interactions between the drug and carrier, ORI is slowly and continuously released from ORI@MOF-5, 
resulting in reduced toxicity. Although ORI@MOF-5 has a weaker killing effect on HepG2 cells than free ORI, its sustained release reduces the toxicity and side effects of drugs, which is more conducive to clinical use.
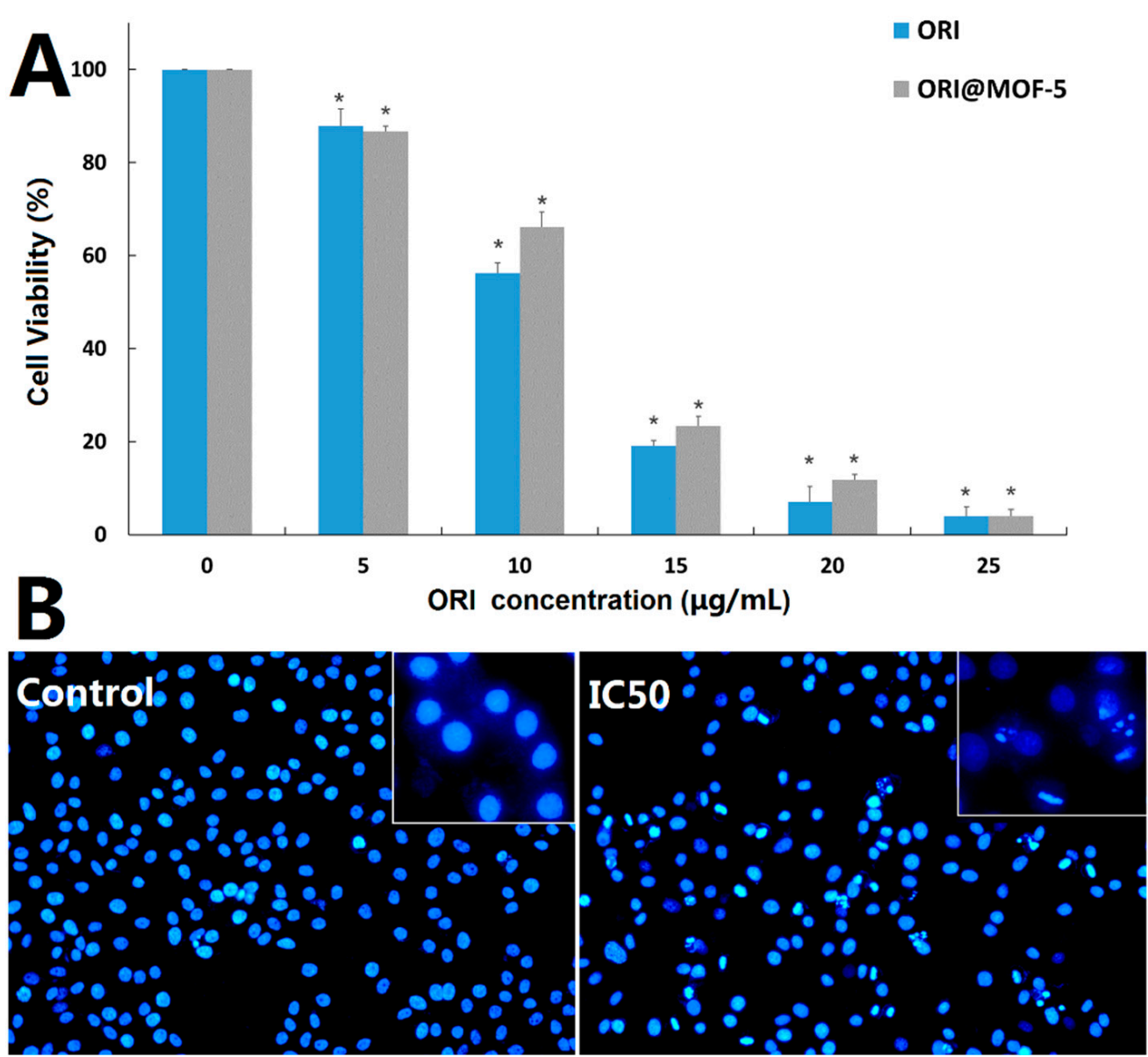

Figure 10. (A) In vitro cell viabilities of HepG2 cells after being incubated for $24 \mathrm{~h}$ with various concentrations of ORI and ORI@MOF-5; (B) Fluorescence microscopic images of HepG2 cells stained with DAPI after 24-h treatment with ORI@MOF-5 (at IC50 value).

After treatment with ORI@MOF-5 at a concentration of 22.99 ug/mL (IC50) for 24 h, HepG2 cells were stained with DAPI. As shown in Figure 10B, compared with the control group, the nuclei of the experimental group occur significantly fragmentation and morphological change, with the common morphological characteristics of apoptosis.

Moreover, the effect of ORI@MOF-5 on apoptosis of HepG2 cells was investigated by Annexin V/PI double staining assay. As illustrated in Figure 11, after treatment with different concentrations of ORI@MOF-5 (containing ORI 0-25 $\mu \mathrm{g} / \mathrm{mL}$ ) for 24h, the apoptosis of HepG2 cells increases with the increase of concentration, which is consistent with the MTT results. Apoptotic cells account for $94.97 \%$ at the highest dose, indicating that ORI@MOF-5 has good anti-tumor effect. 

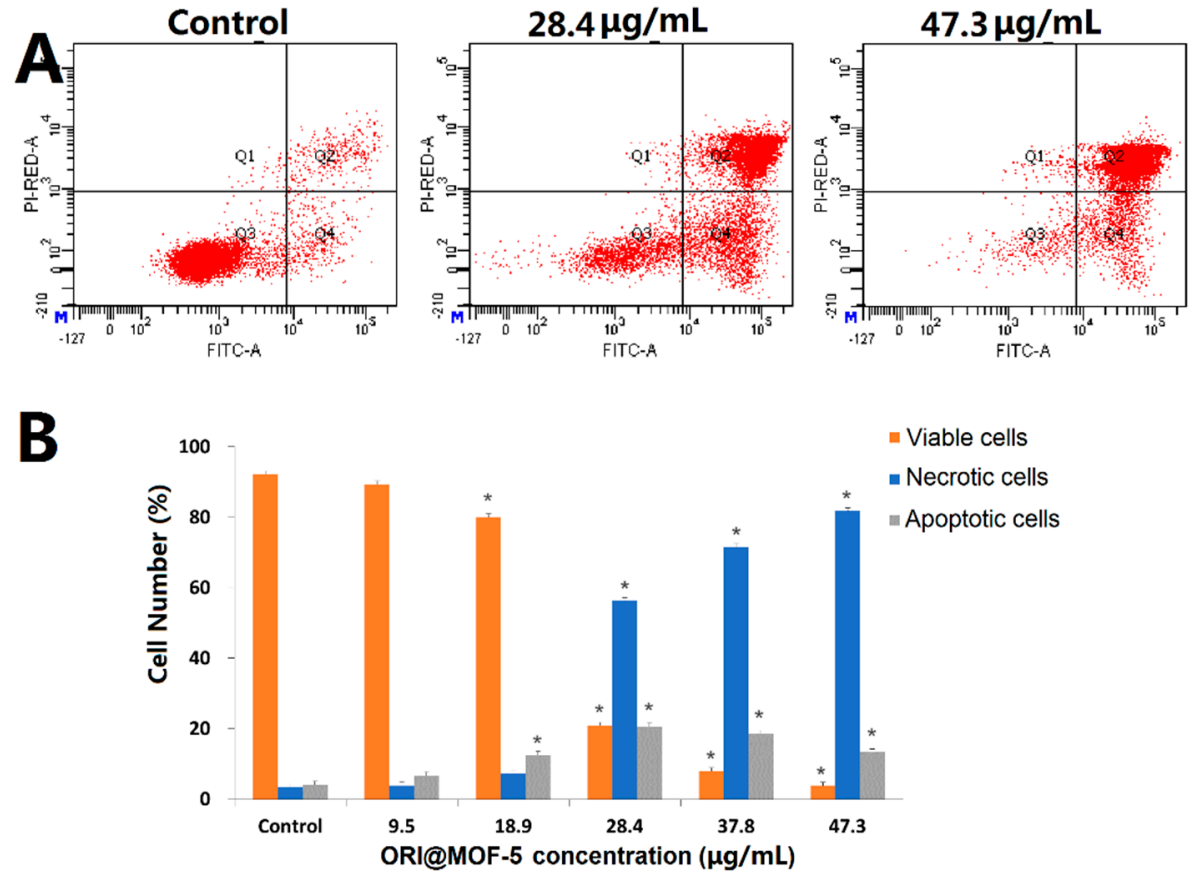

Figure 11. (A) Apoptosis assays for HepG2 cells after treatment with different concentrations of ORI@MOF-5 for 24 h; (B) Statistical analysis of viable, necrotic, and apoptotic HepG2 cells.

\section{Discussion}

In this study, MOF- 5 was selected as the carrier of the antitumor active ingredient ORI for the first time. ORI@MOF-5, a nano-scale sustained release drug delivery system, has been successfully prepared. It has superior biocompatibility, high drug loading, good sustained release effect and excellent anti-tumor effect.

Here, nano-MOF-5 was successfully synthesized by direct addition method, which has a stable structure, good crystal shape and good thermal stability. These are consistent with the results of Huang L, et al. [39]. Compared with the micron-sized MOF-5 synthesized by solvothermal method, we found that MOF- 5 synthesized by direct addition method had better drug-loading effect on ORI. MOF-5-D has small size, high specific surface area and pore volume, which promote drug inclusion. At present, no scholars have conducted research in this area.

By comparing the results of IR, TG, XRD, and BET analysis before and after drug loading, it was found that ORI was successfully loaded into MOF-5. The results of IR showed that the drug-loaded materials contained common characteristic peaks of blank materials and free drugs. XRD results show that the crystallinity of ORI@MOF- 5 is good, and the strong peak at $8.8^{\circ}$ is the combination of carbonyl group in ORI and the metal center in MOF- 5 . At the same time, the specific surface area and porosity of the material decreased significantly after loading, which indicated the loading of the drug.

Then, drug-loading and in vitro release were performed. The drug loading of ORI@MOF-5 is as high as $52.86 \% \pm 0.59 \%$, which is much higher than other types of drug delivery systems, such as gold nanoparticles [46] or graphene oxides [47]. In our previous study [48], ORI was loaded by MIL-53 (Fe), and the drug loading was less than 50\% under the same calculation method. Clinically, high drug loading can improve the efficiency of treatment and reduce the dosage of drug, which is of great significance.

The results showed that ORI@MOF-5 has a nice sustained release effect under different $\mathrm{pH}$ conditions. Its release is basically not affected by $\mathrm{pH}$, and the cumulative release is $87 \%$ in $60 \mathrm{~h}$. This characteristic is linked to the absence of exposed groups in the carrier material itself, which indicates that the drug delivery system can adapt to a variety of $\mathrm{pH}$ environments and is easier to design in clinical use. 
Good biocompatibility is the foundation of the application of carrier materials. In our previous study [49], MTT, DAPI staining, and an apoptosis experiment were used to detect the in vitro toxicity of MIL-100 (Fe), which could reflect the toxicity of the drug comprehensively. Here, the safety evaluation results show that the safe dose of MOF- 5 reaches $25 \mu \mathrm{g} / \mathrm{mL}$, which has little effect on cell viability, nuclear morphology, and cell apoptosis under safe doses. Subsequent experiments were conducted under safe doses.

The efficacy of the drug-loading system determines the value of its clinical value. Compared to free ORI, ORI@MOF-5 has a slightly lower efficacy at the same dose. A similar situation has been reported in the literature [50]. Despite the fact that the drug effect is slightly lower, its sustained release property plays an important role in reducing toxicity and side effects and has more clinical value.

In this study, only the $24 \mathrm{~h}$ toxicity study was performed because after $24 \mathrm{~h}$, the release rate was slow and the cumulative release was slightly below the maximum. What's more, the maximum concentration of cell survival was observed to be less than $10 \%$ at $24 \mathrm{~h}$. Therefore, a longer investigation was not carried out. The characteristics of ORI@MOF-5 drug-loading system are studied only through in vitro experiments, which is not a substitute for in vivo experiments. However, as a conventional evaluation method, it can be used as a basis for future in vivo experiments. In the next step, more different cell lines will be used and animal experiments will be carried out to study the safety, release characteristics, antineoplastic efficacy and other research in vivo, so as to provide better reference for clinical applications.

\section{Conclusions}

In the current study, nano-MOF-5 was synthesized and loaded with the anti-tumor active ingredient ORI. The results show that nano-MOF- 5 has good biocompatibility and biodegradability. Drug-loading and in vitro release experiments confirm that ORI@MOF-5 has high drug loading and good sustained release properties. MTT assay, DAPI staining and apoptosis experiments suggest that ORI@MOF-5 has significant cytotoxicity and apoptosis effect on HepG2 cells. As a result, ORI@MOF-5 has the potential to become a new anti-cancer sustained release preparation.

Supplementary Materials: The following are available online at http://www.mdpi.com/1420-3049/24/18/3369/s1 . Figure S1: High magnification SEM image of MOF-5-S; Figure S2: High magnification SEM image of MOF-5-D.

Author Contributions: X.Y. and J.N. designed the research. G.C., J.L., M.C., L.Q., and Y.D. performed the experiments. Y.W., X.D., and L.G. conducted the data analyses. G.C., P.H., and Y.Y. wrote the paper. All authors have reviewed and approved the final version of the manuscript.

Funding: This research was funded by the Beijing Natural Science Foundation (7194289), the National Natural Science Foundation of China (No. 81703715), the Training Programme Foundation for the Beijing Municipal Excellent Talents (No. 2017000020124G295) and the fundamental Research Funds for the Central Universities (Beijing University of Chinese Medicine Scientific Research Project for Distinguished Young Scholar (No. 2018-JYB-XJQ005). and The APC was funded by the National Natural Science Foundation of China (No. 81703715).

Acknowledgments: This work was financially supported by the Beijing Natural Science Foundation (7194289), the National Natural Science Foundation of China (No. 81703715), the Training Programme Foundation for the Beijing Municipal Excellent Talents (No. 2017000020124G295) and the fundamental Research Funds for the Central Universities (Beijing University of Chinese Medicine Scientific Research Project for Distinguished Young Scholar (No. 2018-JYB-XJQ005).

Conflicts of Interest: The authors declare no conflict of interest.

\section{References}

1. Fitzmaurice, C.; Akinyemiju, T.F.; Al Lami, F.H.; Alam, T.; Alizadeh-Navaei, R.; Allen, C.; Alsharif, U.; Alvis-Guzman, N.; Amini, E.; Anderson, B.O.; et al. Global, regional, and national cancer incidence, mortality, years of life lost, years lived with disability, and disability-adjusted life-years for 29 cancer groups, 1990 to 2016: A systematic analysis for the global burden of disease study. JAMA Oncol. 2018, 4, 1553-1568. [PubMed] 
2. Bray, F.; Ferlay, J.; Soerjomataram, I.; Siegel, R.L.; Torre, L.A.; Jemal, A. Global Cancer Statistics 2018: GLOBOCAN Estimates of Incidence and Mortality Worldwide for 36 Cancers in 185 Countries. CA Cancer J. Clin. 2018, 68, 394-424. [CrossRef] [PubMed]

3. Li, S.; Shi, D.; Zhang, L.; Yang, F.; Cheng, G. Oridonin enhances the radiosensitivity of lung cancer cells by upregulating Bax and downregulating BCL 2 . Exp. Ther. Med. 2018, 16, 4859-4864. [PubMed]

4. Zheng, W.; Zhou, C.-Y.; Zhu, X.-Q.; Wang, X.-J.; Li, Z.Y.; Chen, X.-C.; Chen, F.; Che, X.-Y.; Xie, X. Oridonin enhances the cytotoxicity of 5-FU in renal carcinoma cells by inducting necroptotic death. Biomed. Pharmacother. 2018, 106, 175-182. [CrossRef] [PubMed]

5. Cheng, W.; Huang, C.; Ma, W.; Tian, X.; Zhang, X. Recent Development of Oridonin Derivatives with Diverse Pharmacological Activities. Mini-Rev. Med. Chem. 2019, 19, 114-124. [CrossRef] [PubMed]

6. Seo, E.J.; Fischer, N.; Efferth, T. Phytochemicals as inhibitors of NF-kB for treatment of Alzheimer's disease. Pharmacol. Res. 2018, 129, 262-273. [CrossRef] [PubMed]

7. Hyejin, P.; Ye, J.; Na-Kyung, H.; Joong, K.; Hae-June, L. Oridonin Enhances Radiation-Induced Cell Death by Promoting DNA Damage in Non-Small Cell Lung Cancer Cells. Int. J. Mol. Sci. 2018, 19, 2378.

8. Cui, Q.; Tashiro, S.I.; Onodera, S.; Minami, M.; Ikejima, T. Autophagy Preceded Apoptosis in Oridonin-Treated Human Breast Cancer MCF-7 Cells. Biol. Pharm. Bull. 2007, 30, 859-864. [CrossRef] [PubMed]

9. Zhang, H.P.; Li, G.Q.; Guo, W.Z.; Chen, G.H.; Tang, H.W.; Yan, B.; Li, J.; Zhang, J.K.; Wen, P.H.; Wang, Z.H. Oridonin synergistically enhances JQ1-triggered apoptosis in hepatocellular cancer cells through mitochondrial pathway. Oncotarget 2017, 8, 106833-106843. [CrossRef]

10. Liu, Y.; Liu, J.; Liang, J.; Zhang, M.; Li, Z.; Wang, Z.; Dang, B.; Feng, N. Mucosal transfer of wheat germ agglutinin modified lipid-polymer hybrid nanoparticles for oral delivery of oridonin(Article). Nanomed. Nanotechnol. Biol. Med. 2017, 13, 2219-2229. [CrossRef]

11. Ke, Z.; Zhang, Z.; Wu, H.; Jia, X.; Wang, Y. Optimization and evaluation of Oridonin-loaded Soluplus ${ }^{\circledR}$-Pluronic P105 mixed micelles for oral administration. Int. J. Pharm. 2017, 518, 193-202. [CrossRef] [PubMed]

12. Ding, Y.; Ding, C.; Ye, N.; Liu, Z.; Wold, E.A.; Chen, H.; Wild, C.; Shen, Q.; Zhou, J. Discovery and development of natural product oridonin-inspired anticancer agents. Eur. J. Med. Chem. 2016, 122, 102-117. [CrossRef] [PubMed]

13. Kassem, A.M.; Ibrahim, H.M.; Samy, A.M. Development and optimisation of atorvastatin calcium loaded self-nanoemulsifying drug delivery system (SNEDDS) for enhancing oral bioavailability: In vitro and in vivo evaluation. J. Microencapsul. 2017, 34, 319-333. [CrossRef] [PubMed]

14. Chai, D.D.; Hao, B.J.; Hu, R.; Zhang, F.; Yan, J.; Sun, Y.; Huang, X.Y.; Zhang, Q.X.; Jiang, H. Delivery of Oridonin and Methotrexate via PEGylated Graphene Oxide. ACS Appl. Mater. Interfaces 2019, 11, 22915-22924. [CrossRef] [PubMed]

15. Zhang, X.; Zhang, T.; Lan, Y.; Wu, B.; Shi, Z. Nanosuspensions Containing Oridonin/HP- $\beta$-Cyclodextrin Inclusion Complexes for Oral Bioavailability Enhancement via Improved Dissolution and Permeability. Aaps. Pharmscitech. 2016, 17, 400-408. [CrossRef] [PubMed]

16. Horcajada, P.; Gref, R.; Baati, T.; Allan, P.K.; Maurin, G.; Couvreur, P.; Férey, G.; Morris, R.E.; Serre, C. Metal-organic frameworks in biomedicine. Chem. Rev. 2011, 112, 1232-1268. [CrossRef] [PubMed]

17. Roth Stefaniak, K.; Epley, C.C.; Novak, J.J.; McAndrew, M.L.; Cornell, H.D.; Zhu, J.; McDaniel, D.K.; Davis, J.L.; Allen, I.C.; Morris, A.J. Photo-triggered release of 5-fluorouracil from a MOF drug delivery vehicle. Chem. Commun. 2018, 54, 7617-7620. [CrossRef]

18. Dolgopolova, E.A.; Rice, A.M.; Martin, C.R.; Shustova, N.B. Photochemistry and photophysics of MOFs: Steps towards MOF-based sensing enhancements. Chem. Soc. Rev. 2018, 47, 4710-4728. [CrossRef]

19. Zou, K.Y.; Li, Z.X. Controllable Syntheses of MOF-Derived Materials. Chemistry 2018, 24, 6506-6518. [CrossRef]

20. Zhang, H.; Liu, X.; Wu, Y.; Guan, C.; Cheetham, A.K.; Wang, J. MOF-derived nanohybrids for electrocatalysis and energy storage: Current status and perspectives. Chem. Commun. 2018, 54, 5268-5288. [CrossRef]

21. He, C.; Liu, D.; Lin, W. Nanomedicine applications of hybrid nanomaterials built from metal-ligand coordination bonds: Nanoscale metal-organic frameworks and nanoscale coordination polymers. Chem. Rev. 2015, 115, 11079-11108. [CrossRef] [PubMed]

22. Wu, M.X.; Yang, Y.W. Metal-organic framework (MOF)-based drug/cargo delivery and cancer therapy. Adv. Mater. 2017, 29, 1606134. [CrossRef] [PubMed] 
23. Ibrahim, M.; Sabouni, R.; Husseini, G.A. Anti-Cancer Drug Delivery Using Metal Organic Frameworks (MOFs). Curr. Med. Chem. 2017, 24, 193-214. [CrossRef]

24. Chowdhury, M.A. The applications of metal-organic-frameworks in controlled release of drugs. Rev. J. Chem. 2017, 7, 1-22. [CrossRef]

25. Jiang, K.; Zhang, L.; Hu, Q.; Xin, Z.; Jun, Z.; Yuanjing, C.; Yu, Y.; Bin, L.; Guodong, Q. A zirconium-based metal-organic framework with encapsulated anionic drug for uncommonly controlled oral drug delivery. Microporous Mesoporous Mater. 2019, 275, 229-234. [CrossRef]

26. Qasem, N.A.A.; Ben-Mansour, R.; Habib, M.A. An efficient $\mathrm{CO}_{2}$ adsorptive storage using MOF-5 and MOF-177. Appl. Energy 2018, 210, 317-326. [CrossRef]

27. Li, H.; Eddaoudi, M.; O'Keeffe, M.; Yaghi, O.M. Design and synthesis of an exceptionally stable and highly porous metal-organic framework. Nature 1999, 402, 276. [CrossRef]

28. Sabo, M.; Henschel, A.; Frode, H.; Klemmb, E.; Kaskel, S. Solution infiltration of palladium into MOF-5: Synthesis, physisorption and catalytic properties. J. Mater. Chem. 2007, 17, 3827-3832. [CrossRef]

29. Guo, C.; Zhang, Y.; Zhang, L.; Zhang, Y.; Wang, J. 2-Methylimidazole-assisted synthesis of a two-dimensional MOF-5 catalyst with enhanced catalytic activity for the Knoevenagel condensation reaction. CrystEngComm 2018, 20, 5327-5331. [CrossRef]

30. Bakhtiari, N.; Azizian, S. Nanoporous Carbon Derived from MOF-5: A Superadsorbent for Copper Ions. ACS Omega. 2018, 3, 16954-16959. [CrossRef] [PubMed]

31. Liu, Y.; Ghimire, P.; Jaroniec, M. Copper benzene-1, 3, 5-tricarboxylate (Cu-BTC) metal-organic framework (MOF) and porous carbon composites as efficient carbon dioxide adsorbents. J. Colloid Interface Sci. 2019, 535, 122-132. [CrossRef] [PubMed]

32. Ozen, H.A.; Ozturk, B. Gas separation characteristic of mixed matrix membrane prepared by MOF-5 including different metals. Sep. Purif. Technol. 2019, 211, 514-521. [CrossRef]

33. Cendrowski, K.; Kukulka, W.; Kedzierski, T.; Zhang, S.; Mijowska, E. Poly(vinylidene fluoride) and Carbon Derivative Structures from Eco-Friendly MOF-5 for Supercapacitor Electrode Preparation with Improved Electrochemical Performance. Nanomaterials 2018, 8, 890. [CrossRef] [PubMed]

34. Motakef-Kazemi, N.; Shojaosadati, S.A.; Morsali, A. Evaluation of the effect of nanoporous nanorods $\mathrm{Zn}_{2}(\mathrm{bdc})_{2}$ (dabco) dimension on ibuprofen loading and release. J. Iran. Chem. Soc. 2016, 13, 1205-1212. [CrossRef]

35. Yang, B.; Jiang, Y.; Qin, X.; Chen, Z.; Ren, F. Loaded and in vitro drug release of anticancer drugs in porous metal-organic frameworks. Chem. J. Chin. Univ. 2012, 33, 26-31.

36. Liu, Y.; Wang, Y.; Chen, Y.; Tian, Z.; Xu, C. Study on the adsorption of curcumin by MOF-5 and its digestion in the gastrointestinal tract. J. Food Sci. Technol. 2017, 35, 46-52.

37. Zhang, L.; Chen, Y.; Shi, R.; Rang, T.G.; Pang, G.S.; Wang, B.; Zhao, Y.; Zeng, X.; Zou, C.X.; Wu, P. Synthesis of hollow nanocages MOF-5 as drug delivery vehicle to solve the load-bearing problem of insoluble antitumor drug oleanolic acid (OA). Inorg. Chem. Commun. 2018, 96, 20-23. [CrossRef]

38. Karimzadeh, Z.; Javanbakht, S.; Namazi, H. Carboxymethylcellulose/MOF-5/Graphene oxide bio-nanocomposite as antibacterial drug nanocarrier agent. Bioimpacts 2019, 9, 5-13. [CrossRef]

39. Huang, L.; Wang, H.; Chen, J.; Wang, Z.B.; Sun, J.Y.; Zhao, D.Y.; Yan, Y.S. Synthesis, morphology control, and properties of porous metal-organic coordination polymers. Microporous Mesoporous Mater. 2003, 58, 105-114. [CrossRef]

40. Gao, S.; Zhao, N.; Shu, M.; Che, S. Palladium nanoparticles supported on MOF-5: A highly active catalyst for a ligand and copper-free Sonogashira coupling reaction. Appl. Catal. A Gen. 2010, 388, 196-201. [CrossRef]

41. Eddaoudi, M. Systematic Design of Pore Size and Functionality in Isoreticular MOFs and Their Application in Methane Storage. Science 2002, 295, 469-472. [CrossRef] [PubMed]

42. Burgaz, E.; Erciyes, A.; Andac, M.; Andac, O. Synthesis and characterization of nano-sized metal organic framework-5 (MOF-5) by using consecutive combination of ultrasound and microwave irradiation methods. Inorg. Chimica Acta 2019, 485, 118-124. [CrossRef]

43. Lv, Y.; Yu, H.; Xu, P.; Xu, J.; Li, X. Metal organic framework of MOF-5 with hierarchical nanopores as micro-gravimetric sensing material for aniline detection. Sens. Actuators B Chem. 2018, 256, 639-647. [CrossRef] 
44. Fan, L.; Ge, H.; Zou, S.; Xiao, Y.; Wen, H.H.; Li, Y.; Feng, H.; Nie, M. Sodium alginate conjugated graphene oxide as a new carrier for drug delivery system. Int. J. Biol. Macromol. 2016, 93, 582-590. [CrossRef] [PubMed]

45. Javanbakht, S.; Namazi, H. Doxorubicin loaded carboxymethyl cellulose/graphene quantum dot nanocomposite hydrogel films as a potential anticancer drug delivery system. Mater. Sci. Eng. C 2018, 87, 50-59. [CrossRef] [PubMed]

46. Qiu, W.; Chen, R.; Chen, X.; Zhang, H.; Song, L.; Cup, W.; Zhang, J.; Ye, D.; Zhang, Y.; Wang, Z. Oridonin-loaded and GPC1-targeted gold nanoparticles for multimodal imaging and therapy in pancreatic cancer. Int. J. Nanomed. 2018, 13, 6809. [CrossRef] [PubMed]

47. Jiang, J.H.; Pi, J.; Jin, H.; Cai, J.Y. Functional graphene oxide as cancer-targeted drug delivery system to selectively induce oesophageal cancer cell apoptosis. Artif. Cells Nanomed. Biotechnol. 2018, 46, S297-S307. [CrossRef] [PubMed]

48. Leng, X.; Dong, X.; Wang, W.; Sai, N.; Yang, C.; You, L.; Huang, H.; Yin, X.; Ni, J. Biocompatible Fe-Based Micropore Metal-Organic Frameworks as Sustained-Release Anticancer Drug Carriers. Molecules 2018, 23 , 2490. [CrossRef]

49. Chen, G.; Leng, X.; Luo, J.; You, L.; Qu, C.; Dong, X.; Huang, H.; Yin, X.; Ni, J. In Vitro Toxicity Study of a Porous Iron (III) Metal-Organic Framework. Molecules 2019, 24, 1211. [CrossRef]

50. Javanbakht, S.; Pooresmaeil, M.; Namazi, H. Green one-pot synthesis of carboxymethylcellulose/Zn-based metal-organic framework/graphene oxide bio-nanocomposite as a nanocarrier for drug delivery system. Carbohydr. Polym. 2019, 208, 294-301. [CrossRef]

Sample Availability: Samples of the compounds are not available from the authors.

(C) 2019 by the authors. Licensee MDPI, Basel, Switzerland. This article is an open access article distributed under the terms and conditions of the Creative Commons Attribution (CC BY) license (http://creativecommons.org/licenses/by/4.0/). 